\title{
Poly(2-dimethylamino ethylmethacrylate)-Based Polymers To Camouflage Red Blood Cell Antigens
}

\author{
Bernardino Isaac Cerda-Cristerna, ${ }^{\dagger \dagger}$ Sophie Cottin, ${ }^{\dagger}$ Luca Flebus, ${ }^{\dagger}$ Amaury Pozos-Guillén, ${ }^{\ddagger}$
}

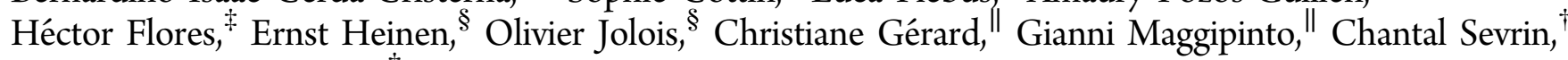
and Christian Grandfils ${ }^{*}{ }^{\dagger}$

\begin{abstract}
${ }^{\dagger}$ Interfacultary Research Center of Biomaterials, University of Liège, Institute of Chemistry, Building B6C, Sart-Tillman (Liège), Liège 4000, Belgium

${ }^{\ddagger}$ Laboratory of Basic Sciences, University of San Luis Potosí, Faculty of Dentistry, Av. Dr. Manuel Nava No. 2, San Luis Potosí, 78290, San Luis Potosí, México

${ }^{\S}$ Biomedical and Preclinical Sciences Department, Human Histology Laboratory, University of Liège, Avenue de l'Hôpital 1, Liège 4000, Belgium

"Hematology and Immuno-Hematology Department, Central Hospital of the University of Liège, Avenue de l'Hôpital 1, Liège 4000, Belgium
\end{abstract}

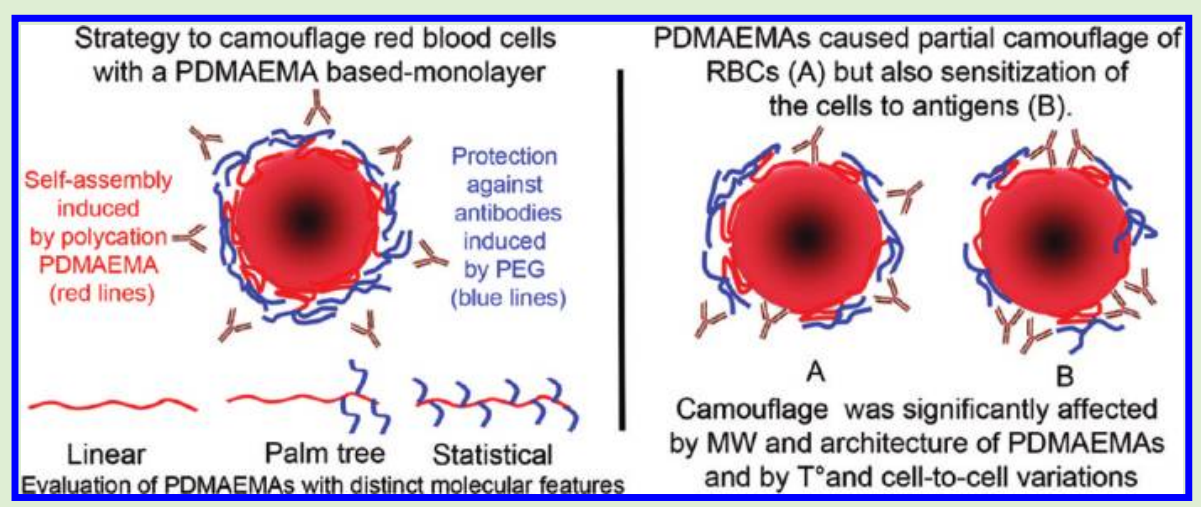

ABSTRACT: Poly(2-dimethylamino-ethylmethacrylate) (PDMAEMA) is a cationic polymer when dissolved in a $7.4 \mathrm{pH}$ fluid. Owing to its ionic nature, this polycation interacts with the negatively charged cell membrane surface of red blood cells (RBCs). The electrostatic self-assembly of PDMAEMA on RBCs membrane can be employed for inducing the formation of a polymeric shield camouflaging blood group antigens on RBCs as a valuable strategy for developing "universal RBCs" for blood transfusion. The purpose of this research was to evaluate the camouflaging ability of PDMAEMA homopolymers and PDMAEMA-copoly(ethylene glycol) copolymers differing in molecular weight and architecture. Surprisingly, the PDMAEMAs caused a partially masking, no masking, and sensitization of the same RBCs population. The MW and architecture of the polymers as well as temperature of PDMAEMA-RBCs treatment influenced the results observed. Herein, the very particular reactivity of PDMAEMAs and RBCs is analyzed and discussed.

\section{INTRODUCTION}

Cell surface engineering is a promising strategy for the production of immunologically silent cells. ${ }^{1,2}$ Accordingly, if cell plasma membranes are physicochemically modified to mask or remove antigens, then cell immunogenicity will be reduced or will totally disappear. ${ }^{1}$ Cell surface modification is a useful approach for the most common type of cell transplantation, in particular, for the transfusion of red blood cells (RBCs; erythrocytes). Transplantation of erythrocytes is governed by the main blood group antigens present on the cell membrane, the $\mathrm{ABH}(\mathrm{ABO}$ system) and $\mathrm{D}$ (Rh system) being the most clinically significant. ${ }^{3,4}$

Because of blood grouping, transfusion requires mandatory matching between blood donor and receptor to avoid any possibility of antigen-antibody response that would induce an immune-hemolytic reaction. ${ }^{5}$ However, although transfusion laboratories perform highly reliable matching tests, mismatching can still occur (5.0 to 5.2 mismatches per 100000 transfusions $\left.{ }^{6}\right)$; even if this inconvenience is prevented by the use of type O RBCs, supplies of these cells are usually insufficient in hospitals. ${ }^{7}$ To sort out this lack of universal RBCs, cell surface modification has become a promising strategy for production of silent RBCs. ${ }^{1,8}$

Received: January 24, 2012

Revised: $\quad$ March 11, 2012

Published: March 14, 2012 
Surface modification to produce silent RBCs has been explored with two major approaches: the enzymatic cleavage of antigens and the camouflaging of antigens by polymers. ${ }^{1}$ The former relies on enzymatic cleavage of the monosaccharide $A$ or $\mathrm{B}$ antigenic sites to convert RBCs into type $\mathrm{O}$ cells (ECORBCs). ${ }^{9,10}$ Although enzymatic cleavage has significantly reduced the antigenic properties of RBCs, 9,10 ECO-RBCs have still shown reactivity to antibodies, ${ }^{1,10}$ but the main drawbacks of this method are the difficulty of large-scale and high cost of production of the requested enzymes.

The other approach involves the formation of a polymeric shield around RBCs by either covalent or electrostatic binding of polymers. Covalent binding has been studied especially by grafting poly(ethylene glycol) (PEG) polymer. This nonionic polymer is largely used for RBC surface modification because of its flexible structure and physicochemical properties that favor the formation of a water-polymeric shield-inducing steric repulsion of antibodies. ${ }^{111}$ However, although PEGylated RBCs have been studied intensively, concerns still exist about the total efficacy of PEG to mask cell antigens and due to the rapid clearance of the PEGylated RBCs. ${ }^{1}$ Another method of forming a polymer shield relies on the self-assembly of cationic polymers on the RBC surface, ${ }^{12,13}$ taking advantage of negative charges on the carbohydrate structures on the RBC surface, including those on antigens. Polymeric self-assembly by layerby-layer (LbL) method has also been used to grow polyelectrolyte layers to modify RBCs. When the LbL method is applied on the erythrocyte surface, a polycation-based layer is formed first, then the excess of free positive charges is used to adsorb a subsequent anionic-based layer; this process can be repeated to increase the thickness of the coating. This method has been reported to adopt a double layer of chitosan- $g$ phosphorylcholine-sodium hyaluronate and poly-L-lysine (PLL)-g-PEG-sodium alginate in view to block anti-A antibodies. ${ }^{12,13}$ Even though LbL is promising, it may increase the risk of altering the membrane properties, in particular, their flexibility. In this respect, a single polymer layer made by a simple one-step method, as proposed by Elbert and Hubell, is an attractive alternative. These authors have performed selfassembly of PLL- $g$-PEG on RBCs blocking lectin-induced hemagglutination. ${ }^{14}$ Herein, using self-assembly, we have investigated poly(2-dimethylamino-ethymethacrylate) (PDMAEMA)-based polymers to camouflage RBC antigens. The PDMAEMA sequence, partially cationized at $\mathrm{pH} 7.4$, is expected to anchor the synthetic macromolecule on the RBC surface. These polymers include PEG grafts to form a steric barrier, promote antibody repulsion, and avoid cell aggregation. Utilizing this polymer family, Riquelme et al. have already reported that PDMAEMA-co-MAPEG copolymers (MW 29100 and 30700 ) did not alter the morphological and rheological properties of RBCs up to a polycation concentration of $250 \mu \mathrm{g} / \mathrm{mL}^{15}$ Moreover, those polymers induced partial masking of antigens A and B and of glycophorin A (GPA). Additionally, a previous hemocompatibility study of PDMAEMAs with whole human blood has shown their safety up to $100 \mu \mathrm{g} / \mathrm{mL}^{16}$ However, there is still a lack of understanding of PDMAEMA-RBC interaction and the effect of PDMAEMAs on RBC camouflage. In the present study, we assessed the immune-camouflaging ability of PDMAEMA homopolymers of differing MWs. Their properties have been compared with three distinct PDMAEMA-co-MAPEG copolymers to better understand the correlation between the macromolecular features of these polymers and their effect on antigen masking. Immunomasking was evaluated by adopting fluorescent antiglycophorin A antibody and using flow cytometry to quantify cell immunoprotection considering different polymer concentrations and performing the polymeric coating at temperatures ranging between 25 and $37^{\circ} \mathrm{C}$.

\section{MATERIALS AND METHODS}

2.1. Materials. 2-(Dimethylamino) ethyl methacrylate (DMAEMA) and PEG $\alpha$-methoxy, $\omega$-methacrylate (MAPEG), Drabkin's reagent (cyanmethemoglobin), Brij 35, and bovine hemoglobin were purchased from Sigma-Aldrich (Bornem, Belgium). Fluorescein isothiocyanate (FITC) mouse monoclonal antibody (Mouse IgG1) antihuman GPA (CD235a) (aGPA) was purchased from Invitrogen (Merelbeke, Belgium). Phosphate-buffered saline (PBS) pH 7.4 was prepared with $\mathrm{KH}_{2} \mathrm{PO}_{4}, 1.4 \mathrm{mM} ; \mathrm{Na}_{2} \mathrm{HPO}_{4}, 10 \mathrm{mM}$; NaCl, $137 \mathrm{mM}$; and $\mathrm{KCl}, 2.7 \mathrm{mM}$. All other chemicals and reagents were of analytical grade.

2.2. Synthesis and Characterization of PDMAEMA-Based Polymers. The PDMAEMA-based polymers were synthetized by a solvent-free, atom-transfer radical polymerization (ATRP) method. Synthesis, characterization, and purification of polymers were performed as reported elsewhere. ${ }^{16}$ After purification, each polymer was dissolved in PBS (stock solution $1 \mathrm{mg} / \mathrm{mL}$ ) and stored at $-20{ }^{\circ} \mathrm{C}$ until their use for the experiments.

2.3. Blood Collection and RBC Suspension. Type A blood group samples were employed to avoid any variation between blood groups. Blood samples were originated from healthy donors of the Red Cross Blood Transfusion Department in The Central Hospital of the University of Liège. Blood was collected in $3.2 \%$ citrated $4.5 \mathrm{~mL}$ tubes. After collection, blood was centrifuged for $10 \mathrm{~min}$ at $3000 \mathrm{rpm}$ to separate the RBCs from the plasma. The erythrocytes were washed three times with PBS under the same conditions of centrifugation. Finally, a $5 \% \mathrm{RBC}\left(\sim 6.8 \times 10^{5} \mathrm{RBCs} / \mu \mathrm{L}\right)$ suspension (RBC suspension) was prepared for the experiments. All experiments were done within $2 \mathrm{~h}$ of collection. This study received the approval of the Ethics Committee of the Medical Faculty of Liège.

2.4. Polymeric Coating of the RBCs. In brief, in a $2 \mathrm{~mL}$ cylindrical microcentrifuge polypropylene tube, $150 \mu \mathrm{L}$ of the RBC suspension was added to an equal volume of a polycation solution. The cell suspension was mixed immediately afterward using three upand-down movements to avoid any gradient concentration of the polymer. Immediately after, the cell suspension was incubated under lateral agitation (CAT model M5, CAT Ingenieurbüro, Staufen, DE) at a rate of $36 \mathrm{rpm}$ for $1 \mathrm{~h}$ at $37^{\circ} \mathrm{C}$. This second mixing was performed at room temperature $\left(\mathrm{RT}^{\circ} ; 25^{\circ} \mathrm{C}\right)$ for the homopolymers, whereas for the copolymers, three distinct temperatures $\left(25,30\right.$, and $\left.37^{\circ} \mathrm{C}\right)$ have been investigated. After that, $1 \mathrm{~mL}$ of PBS was added to the treated $\mathrm{RBCs}$ to wash and centrifuge them (Mini Spin Plus Microcentrifuge, Eppendorf) for $10 \mathrm{~min}$ at $600 \mathrm{rpm}$. This step was repeated twice (three washing steps in total). Then, the RBCs were resuspended at $5 \%$ concentration.

2.5. Evaluation of Fluorescence in Treated RBCs. After adding $5 \mu \mathrm{L}$ of aGPA $(4.5 \mu \mathrm{g} / \mathrm{mL})$ to $50 \mu \mathrm{L}$ in a conic Eppendorf tube $(1.5$ $\mathrm{mL}$ ) of either treated or control erythrocyte suspension, the PDMAEMA RBCs and native RBCs (nontreated) were incubated for $30 \mathrm{~min}$ at $4{ }^{\circ} \mathrm{C}$ (lateral agitation, $300 \mathrm{rpm}$ ). After incubation, the cells were gently washed with $1 \mathrm{~mL}$ of PBS and centrifuged at 1200 $\mathrm{rpm}$ for $5 \mathrm{~min}$ at $\mathrm{RT}^{\circ}$ (Mini Spin Plus Microcentrifuge). Because we used an FITC-conjugated antibody, only a washing step was performed. ${ }^{17}$ Samples were analyzed $30 \mathrm{~min}$ after being stored in darkness at RT.

All samples were analyzed with a FACSCalibur flow cytometer at $\mathrm{RT}^{\circ}$ (FSC Volt 800; SSC volt 447; FL1 615, FL2 550, FL3 650) (Becton Dickinson, Erembodegem, Belgium). Collected data were analyzed with WinMDI 2.8 software (Purdue University Cytometry Laboratories, West Lafayette, IN). Native RBCs were used as a sample reference for size distribution and granularity, and the native RBCs incubated with aGPA were used as sample reference for fluorescence (mean fluorescence intensity (MFI)). The experiments were 
performed in triplicate and with at least two repetitions. A one-way ANOVA and Tukey-Kramer HSD were performed to identify possible significant statistical differences between MFI and percentage of RBCs (RBCs number) of PDMAEMA-RBCs and untreated RBCs.

2.6. Evaluation of RBCs by Optical Microscope. When required, the treated $\mathrm{RBCs}$ were observed by an optic microscope as reported by Cerda-Cristerna et al. ${ }^{16}$ In brief, $10 \mu \mathrm{L}$ of PDMAEMARBCs (5\% concentration) was diluted in $990 \mu \mathrm{L}$ of PBS in a $1.5 \mathrm{~mL}$ Eppendorf tube. An aliquot $(40 \mu \mathrm{L})$ of that suspension, dropped into a flat-bottomed, 96-well multiplate, was observed with an inverted microscope (Inverso-TC, CETI) at $\times 25$ magnification and imaged with a digital camera (VisiCam 5.0, VWR, Leuven, Belgium). Two repetitions were performed using distinct polymer batches.

\section{RESULTS AND DISCUSSION}

3.1. Synthesis and Characterization of PDMAEMA. It should be stressed that ATRP has as main advantageous to tailor well-defined PDMAEMA-based polymers for evaluating their coating properties. Control of the molecular features of these macromolecules was particularly important in view to be able to correlate their macromolecular features (architecture, MW, polydispersity) and their hemoreactivity. Table 1 shows

Table 1. Characteristics of the PDMAEMA Homopolymers and Copolymers ${ }^{a}$

\begin{tabular}{|c|c|c|c|c|c|}
\hline code & polymer architecture & $M_{\mathrm{w}}$ & PDI & $\begin{array}{l}\text { PDMAEMA } \\
\text { molar } \%^{b}\end{array}$ & $\begin{array}{l}\text { PEG } \\
\left(M_{\mathrm{n}}\right)\end{array}$ \\
\hline P1 & linear PDMAEMA & 10000 & 1.19 & 100 & \\
\hline $\mathrm{P} 2$ & linear PDMAEMA & 26400 & 1.17 & 100 & \\
\hline P3 & $\begin{array}{l}\text { poly(DMAEMA-block- } \\
\text { MAPEG) }\end{array}$ & 30000 & 1.20 & 85 & 480 \\
\hline P4 & $\begin{array}{l}\text { poly(DMAEMA-block- } \\
\text { MAPEG) }\end{array}$ & 30700 & 1.18 & 75 & 3050 \\
\hline P5 & $\begin{array}{l}\text { poly(DMAEMA- } \\
\text { random-MAPEG) }\end{array}$ & 29000 & 1.52 & 51 & 480 \\
\hline
\end{tabular}

${ }^{a}$ Relative average MWs $\left(M_{\mathrm{w}}\right.$ and polydispersity index (PDI) were determined by size exclusion chromatography in THF/triethylamine (TEA) (2.5\%) against polystyrene standards. ${ }^{b}$ Molar \% of PDMAEMA was determined by ${ }^{1} \mathrm{H}$ NMR in $\mathrm{CDCl}_{3}$.

the characteristics of the PDMAEMA-based polymers considered in this study. Two linear PDMAEMA homopolymers (coded P1 and P2) were selected in view to assess the influence of the difference in molecular length of the polycation sequence on the masking ability of PDMAEMA on RBCs. Two palm-tree PDMAEMA-co-MAPEG copolymers (coded P3 and P4) and a random PDMAEMA-co-MAPEG copolymer (coded P5) have been investigated to evaluate the steric effect brought by the polyethylene oxide sequence on antigen masking ability. In the case of $\mathrm{P} 3$ and $\mathrm{P} 4$, they have palm-tree architecture, but $\mathrm{P} 4$ has higher PEG content and longer PEG chains than P3. The random P5 has statistically distributed PEG with length identical to P3.

To avoid any side reactivity of the blood with monomer, catalyst, or initiator residues, we paid special attention to purify these polymers extensively, having been submitted to three successive and complementary extraction steps. After purification, $\mathrm{Cu}$ residue, determined by ICP/MS, was found to be 158 \pm 17 ppm. Monomer residue, estimated from ${ }^{1} \mathrm{H}$ NMR analysis, was below $0.1 \%$.

3.2. Effect of PDMAEMA Homopolymers on RBCs. Antigen GPA was selected to evaluate camouflaging because it furnishes most of negative charges of the $\mathrm{RBC}$ membrane and represents a major protein component of the RBC's surface with a number of copies in the range of 1 million/cell with few variation in density compared with the $\mathrm{ABO}$ antigens. ${ }^{18}$ Before considering the copolymers, we were curious to explore the effect of only the PDMAEMA polymer for camouflaging of RBCs and compare it with the copolymers. Indeed, surfaces modified with PDMAEMA have displayed antifouling properties. ${ }^{19}$ This resistance to protein adsorption has been achieved after elimination of electrostatic interactions, thus playing on either ionic strength or $\mathrm{pH}$. For that purpose, we assessed coating the RBCs with either P1 or P2 (P1-RBCs and P2$\mathrm{RBCs}$, respectively), considering two polycation concentrations: 2 and $10 \mu \mathrm{g} / \mathrm{mL}$. aGPA-incubated RBCs as well as native $\mathrm{RBCs}$ were adopted as controls. (See representative dot plots in Figure 1.)

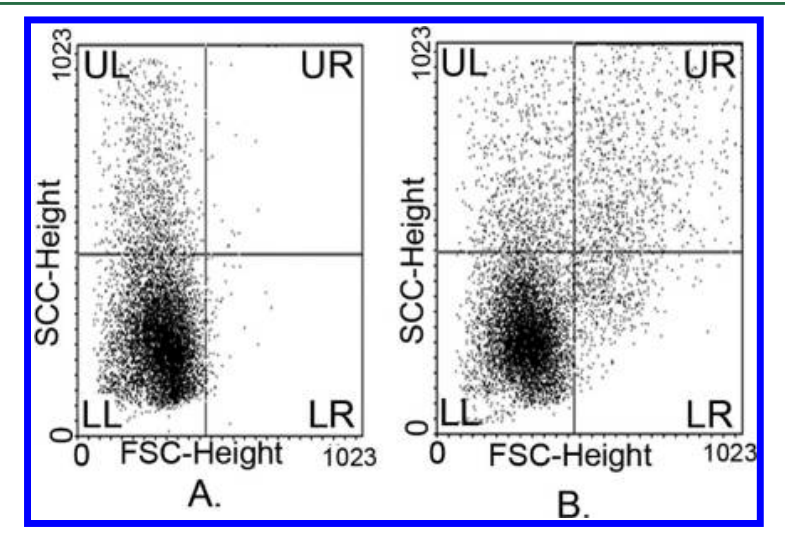

Figure 1. Dot plots, granularity versus size distribution ( $y$ axis vs $x$ axis, respectively) noticed during the analysis of (A) native RBCs and (B) P2 $10 \mu \mathrm{g} / \mathrm{mL}-\mathrm{RBCs}-\mathrm{aGPA}$. The RBCs incubated with only aGPA or with only P1 and P2 presented a RBCs distribution similar to A.

Size distribution and granularity of untreated RBCs have been mainly observed on the lower left [LL] quadrant of the dot plots with $\sim 15 \%$ of events noticed on the upper left [UL] (Figure 1A). A similar cell distribution pattern was also recorded after treatment with $\mathrm{P} 1, \mathrm{P} 2$, or aGPA, but surprisingly enough, when the P2-RBCs were incubated with aGPA, both size distribution and granularity were incremented. This augmentation, noticed on the right side of the dot-plots (upper right [UR] and lower right [LR] quadrants) (Figure 1B), typically results from a cell aggregation.

In view of understanding the origin of cell aggregation mediated by aGPA, we included two additional controls. The first relied on incubating PDMAEMA-RBCs with bovine serum albumin (BSA), a protein present at $4 \mathrm{mg} / \mathrm{mL}$ concentration in the reagent solution of the aGPA. Indeed BSA could potentially act as a cross-linker between RBCs due to its size and global negative charge at neutral $\mathrm{pH}$. If PDMAEMA adsorbed to the RBC membrane, then we would anticipate that its positive domains could interact with the anionic sites of BSA, therefore promoting cell aggregation. We also included another control consisting of PDMAEMA-RBCs with a nonspecific RBC antibody, anti-CD45 (aCD45). This mouse IgG1 antibody, although having macromolecular features similar to aGPA (unlike BSA), is not likely to interact with human RBCs. In the case of positive answer, this response would result from nonspecific interaction and should proceed via FC fragment. For a better and more quantitative interpretation of the results, the percentage of events on the right side of each dot plot was subtracted from the percentage of events on the left side. 
Accordingly, if cell aggregation occurs, then the percentage of RBCs (\% RBCs) on the left side would decrease. Interestingly, the results demonstrated that neither BSA nor aCD45 affected the \% RBCs (Figure 2). This evidence supports the selectivity

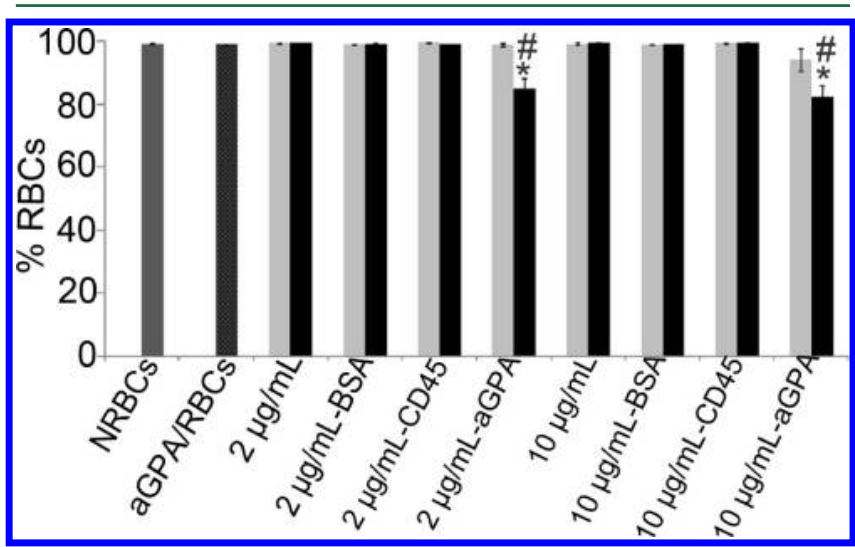

Figure 2. Evolution of the RBC percentages of the left side FACS events after subtraction of right events noticed for RBCs treated with PDMAEMA and incubated with aGPA, BSA, or aGPA. NRBCs: native RBCs; aGPA/RBCs: RBCs incubated with aGPA. Light gray bar: P1treated RBCs; black bar: P2-treated RBCs. *: statistically significant differences between that sample and native RBC. \#: statistically significant differences between P2 and its corresponding P1. \% RBCs of each bar is the average of at least two distinct replicas. of RBC aggregation due to specific GPA recognition. This immune-cell agglutination was corroborated by optical microscopy. As highlighted on Figure 3, the comparison of the microscopic pictures confirms that the specific $\mathrm{RBC}$ agglutination is observed only under the P2-aGPA-RBC conditions.

These observations showed that PDMAMEA with a MW above 10000 and at concentrations of at least $2 \mu \mathrm{g} / \mathrm{mL}$ selectively enhances cell agglutination following immune recognition of the GPA antigens on the RBC surface. As a consequence of the formation of polyelectrolyte complexes between PDMAEMA and anionic sites of RBC's membrane, with as result a reduction of their zeta potential, ${ }^{20,21}$ this unexpected result might be explained the enhancement in immune-specific RBC aggregation. ${ }^{22,23}$ It should be stressed that in the range of PDMAEMA concentration and MW considered in this section, we are far from totally neutralizing the RBCs. We have observed that $\mathrm{P} 1$ at $10 \mu \mathrm{g} / \mathrm{mL}$ reduces the RBCs' electrophoretic mobility from -2.85 to $-2.50 \mu \mathrm{m} \cdot \mathrm{cm} /$ volts without inducing cell aggregation (data not published); a similar effect of P2 can evidently be expected. In addition to the effect of PDMAEMA on the negative charges of RBCs, the aGPA could also participate in the reduction of zeta potential. GPA is a sialoglycoprotein composed of an intracellular, a transmembrane, and an extracellular domain. ${ }^{24}$ Because the latter domain contains a high sialic acid content, GPA is the main source of negative charges representing $\sim 60 \%$ of the RBCs' negative surface charge. ${ }^{24}$ Accordingly, the principal

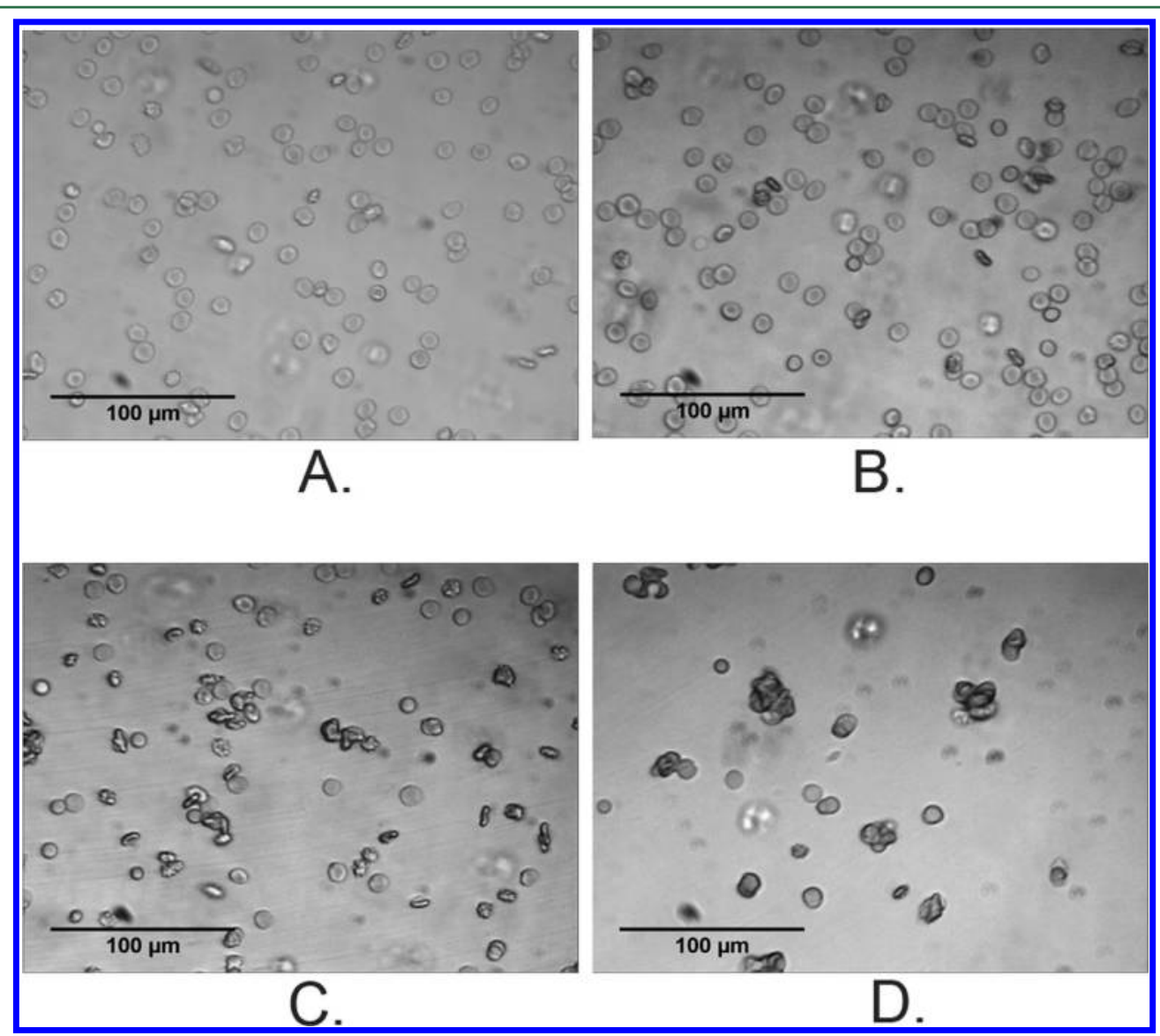

Figure 3. Optical micrographies $(\times 25)$ of RBCs: (A) control sample (Similar observations were done for RBCs treated only with P1 or P2); (B) RBCs treated with aGPA; (C) RBCs treated with P1 $(10 \mu \mathrm{g} / \mathrm{mL})$ and aGPA; and (D) RBCs treated with P2 $(10 \mu \mathrm{g} / \mathrm{mL})$ and aGPA. Bar represents $100 \mu \mathrm{m}$. 
function of GPA is induction of cell-to-cell repulsion, avoiding RBC aggregation. ${ }^{17}$ Taking into account he fact that aGPA is an IgG1 antibody with an isoelectric point in the range of 6.4 to $9.0,25$ its binding to GPA should contribute to neutralization of the RBC membrane under the conditions adopted within our experiment. Moreover, antibody binding may affect the electrical charges of the antigen. ${ }^{25}$

An additional factor that should help to enhance cell aggregation relies on the fact that the partial neutralization of negative surface charges originally expressed by RBCs should also facilitate cell bridging due the double valence of aGPA-IgG. Indeed, a reduction of RBCs zeta potential should promote cell bridging through the interaction of the two binding sites of the IgG molecules with antigens expressed by the two adjacent cells. Therefore, aGPA molecules might act as a cell crosslinkage to promote RBC aggregation. It has been reported that other polycations, such as protamine and polybrene, also promote RBC antibody detection because of their ability to reduce intercellular distance. ${ }^{26,27}$ Although these assumptions will need experimentations to support them, it would be valuable to valorize these observations as a mean of enhancing immunoreaction for diagnostic purposes.

We also assessed the ability of homopolymers to mask the cells. This property has been analyzed based on two main criteria, that is, the percent of immunomasked RBCs in a given population of normal RBCs and the extent of antigen covering/ immunoprotected cell. These two parameters, identified as efficiency and effectiveness, respectively, have been determined from FACS diagrams as typically reported in Figures 4 and $5 A, B$.

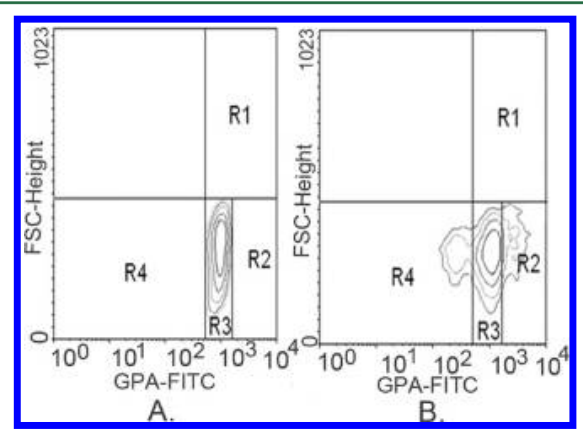

Figure 4. Contour plots showing localization of R1, R2, R3, and R4. The $y$ axis measures fluorescence, and the $x$ axis indicates size distribution. (A) Native RBCs incubated with aGPA. A homogeneous cell population is located in R3. (B) P2-treated RBCs. A nonhomogenous population is observed. All cells located in R4 present partial camouflage.

The native RBCs incubated with aGPA presented a homogeneous cell population showing a signal of fluorescence that was used as a reference point to determine the percentage of camouflaged RBCs. Surprisingly, PDMAEMA-RBCs presented a cell population with distinct regions of fluorescence. To analyze those samples, we determined four clockwise regions (Figure 4) based on the reference point of fluorescence (aGPA/RBCs): Region 1 (R1): events located there were exclusive of the analysis because they represented cell aggregates without value for the interpretation of other data; Region 2 (R2) corresponded to a higher MFI. If PDMAEMARBCs were located there, then they were considered to be sensitized RBCs (sRBCs); Region 3 (R3) presented cells with MFI similar to the control RBCs; therefore, PDMAEMA-RBCs

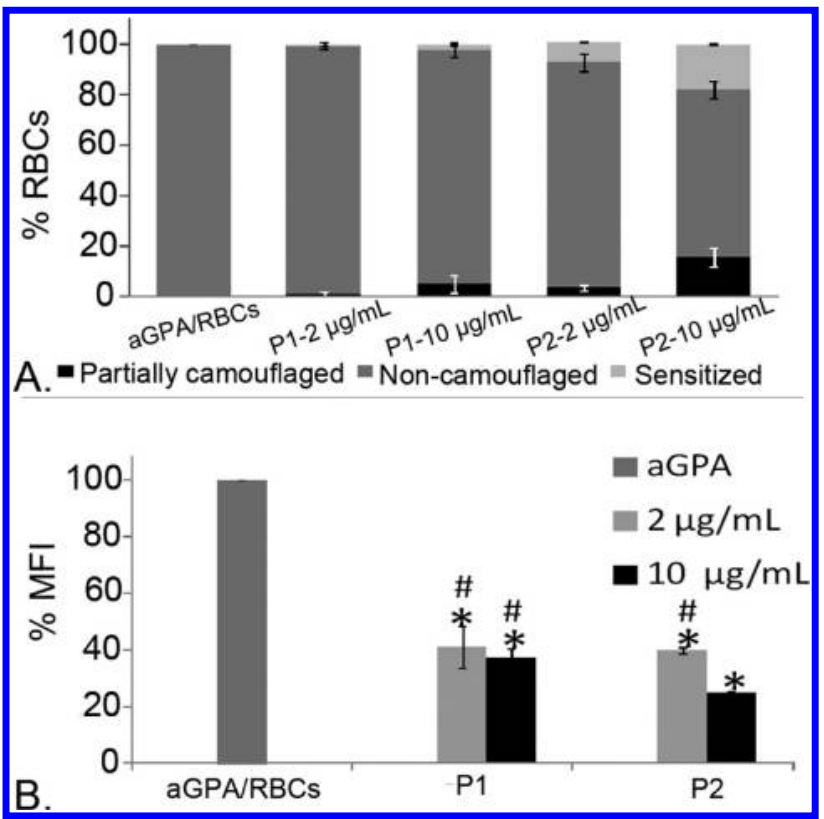

Figure 5. (A) Distribution of percentage of partially camouflaged, noncamouflaged, and sensitized RBCs in a PDMAEMA-RBC population represented by each bar. (B) \% MFI Bars with (*): significantly statistical differences between that sample and native RBCs incubated with aGPA. Bars with (\#): significantly statistical differences between those samples and $\mathrm{P} 2$ at $10 \mu \mathrm{g} / \mathrm{mL}$. A bar is the average of at least two distinct replicas.

located there were considered to be noncamouflaged RBCs (ncfRBCs); and Region 4 (R4): if PDMAEMA-RBCs were present, then they were considered to be camouflaged RBCs.

The percentage of $\mathrm{R} 2, \mathrm{R} 3$, and $\mathrm{R} 4 \mathrm{cfRBCs}$ was determined by the number of events located in each of these regions (Figure 5). For discussion purposes only, we identified the sRBCs, ncfRBCs, and cfRBCs as subpopulations.

Although the camouflage efficiency remained low for all conditions assessed, some dependence was observed as a function of PDMAEMA concentration and MW (Figure 5A). Both an increase in polycation concentration and in MW raised the antigen masking efficiency with a maximum effective protection noticed $(15.4 \pm 3.7 \%$ cfRBCs $)$ for $\mathrm{P} 2$ at $10 \mu \mathrm{g} / \mathrm{mL}$. To evaluate the camouflage extent per cell, that is, the immunomasking effectiveness, we compared the percentage of mean fluorescence intensity ( $\% \mathrm{MFI}$ ) of the protected cells (calculated by reference to the MFI of the native cells incubated with aGPA and considered as $100 \%$ ). This parameter indicated that although most of the blood cells (up to 95\%) kept GPA antigens accessible to the antibody, the protected cells demonstrated a strong reduction in \% MFI (up to $80 \%$ reduction compared with the control cells) (Figure 5B). Although no total camouflaging was observed, this series of experiments highlights interesting findings on the heterogeneity in reactivity of the RBC population to the PDMAEMA's macromolecules.

Before discussing these points, we have estimated whether the number of polycation molecules added to the RBCs was enough to cover the entire surface of the cells. For that calculation, we started from two approximations: (1) 1 MW 20 000 PDMAEMA molecule should cover $75 \mathrm{~nm}^{2}$ of the cell surface (based on the hydrodynamic diameter of PDMAEMA in solution of $10 \mathrm{~nm}$ ), and (2) the area of an RBC is $135 \mu \mathrm{m}^{2}{ }^{28}$ On the basis of these data, full coverage of the RBCs would 
need $\sim 1.7$ million polycation molecules if they adsorb under the form of a regular monolayer at the plasma membrane surface. Considering the concentration of the RBC suspension used for the coating ( $2.5 \%$ when diluted with the polycation solution), we can readily calculate that the two polymer concentrations used (i.e., 2 and $10 \mu \mathrm{g} / \mathrm{mL}$ ) should correspond to 50 and $250 \%$ of the total theoretical surface exposed by the RBCs. Although this calculation is limited by the exact conformation of the polycation macromolecules once adsorbed to the membrane and of their compaction/surface density, the estimate provides an approximate picture of the polyelectrolyte molecules used to cover the cell membrane. This assumption does not take into account the possible condensation of the PDMAEMA upon its adsorption to the cell membrane surface due to the formation of protein-polyelectrolyte complexes with, as a result, a reduction in hydrodynamic diameter of the synthetic polymer with an organization status, which should be affected by the experimental conditions adopted during the coating. ${ }^{29}$ Keeping in mind the limits of our assumption, our experimental conditions should permit completely coating the RBCs' surface with a $10 \mu \mathrm{g} / \mathrm{mL}$ PDMAEMA concentration, considering an association constant favorable for their migration to the surface. In contrast with the apparent lack of reactivity of PDMAEMA to the RBC, the existence of RBCs with an increased percentage of FMI is astonishing.

With respect to PDMAEMA-induced sensitization, it is well known that polycations such as protamine and polybrene enhance RBCs' antibody detection because of reduction in intercellular distance facilitating agglutination. ${ }^{26,30}$ Therefore, polycations act on the second stage of agglutination, that is, agglutination by itself, but they do not influence the first stage, sensitization. ${ }^{26}$ Surprisingly, taking into account the fact that sensitized cells did not increase their size distribution (i.e., cells located at Region 2), PDMAEMA-induced sensitization was not due to cell aggregation. RBC sensitization could occur if the synthetic polymer could promote attraction of aGPA, therefore, through an increase in affinity of the antigen-antibody interactions. From this point of view, it is worth reminding that the antigen and antibody interaction is the result of the nanometric interplay of these two entities with the establishment of long-range ionic bonds and short-range hydrophobic interactions that overcome the hydration energy of the antigen and the antibody. ${ }^{26}$ Obviously it would not be surprising that the precoating of the cell glycocalyx with PDMAEMA could affect this antigen-antibody interplay by modifying at least the local ionic environment and participate in increasing ionic bonds on the RBC surface with aGPA. In addition to participating in the control of ionic bonds on RBCs surface, the hydrophobic and hydrophilic areas of the PDMAEMA backbone, partially protonated at neutral $\mathrm{pH},{ }^{31}$ could also alter the hydrophobicity/hydrophilicity ratio of the cell surface and could promote the physical removal of water molecules to facilitate aGPA binding.

3.3. Effect of PDMAEMA Copolymers on RBCs. Three distinct PDMAEMA-co-MAPEG copolymers (Table 1) were used to assess the ability of the PEG sequence to provide the steric repulsion expected to increase the immunomasking action. This comparison should allow us to explore the influence of both the length of the PEG segments (oligomers of MW 480 or 3050; comparing P3 and P4, respectively) and the PEG distribution along the PDMAEMA backbone (palm tree vs random distribution; comparing P3 and P4 vs P5, respectively).
Riquelme et al. have noticed that those copolymers affected the RBCs and induced aggregation at $125 \mu \mathrm{g} / \mathrm{mL},{ }^{15}$ we decided to assess them at concentration below $100 \mu \mathrm{g} / \mathrm{mL}$. Additionally, taking into account the thermoresponsive properties of PDMAEMA-based polymers, ${ }^{32}$ we compared the effect of three temperatures $\left(25^{\circ} \mathrm{C}, 30^{\circ} \mathrm{C}\right.$, and $\left.37^{\circ} \mathrm{C}\right)$ on immunomasking. To facilitate the comparison of polycations hemoreactivity, we integrated within Figure 6 the evolution of the percentages of

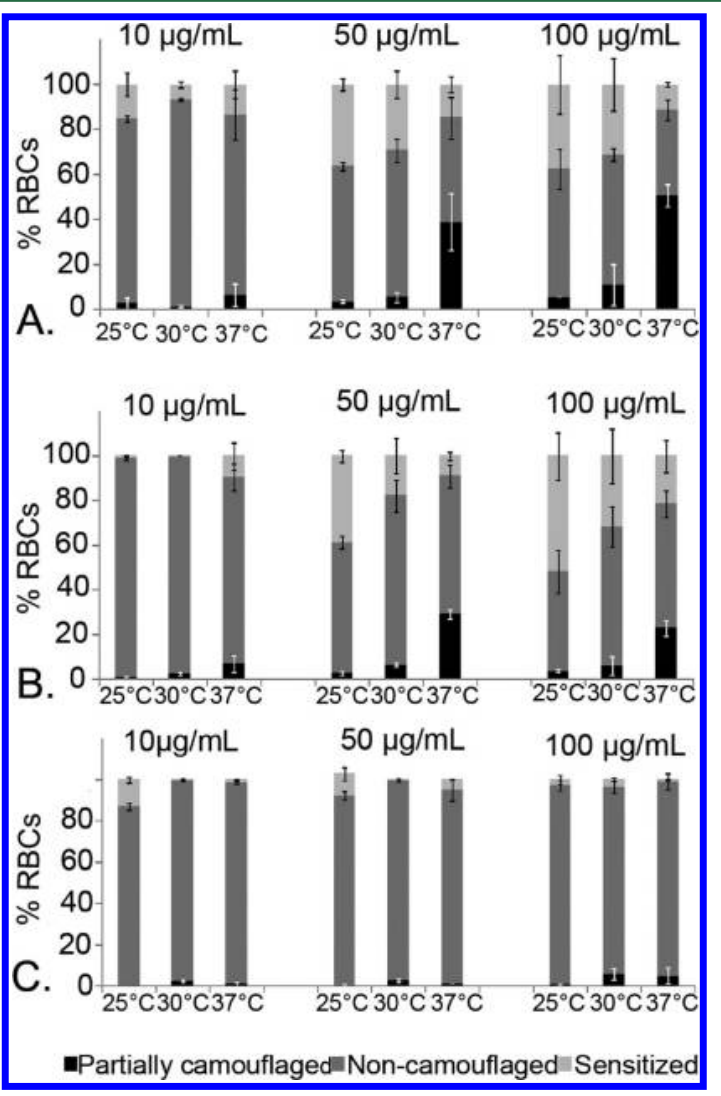

Figure 6. Effect of temperature, nature, and concentration of polycations on their immunomasking efficiency. The percentages of pcfRBCs, ncRBCs, and sRBCs in a PDMAEMA-RBC population are represented by each bar. (A) P3-treated samples, (B) P4-treated samples, and (C) P5-treated samples.

pcfRBCs, ncfRBCs, and sRBCs as a function of the three variables, that is, nature and concentration of the copolymers and temperature. From these data, it is straightforward to notice that P5 has a behavior totally different from the other copolymers but also compared with the homopolymers. Indeed, whatever the conditions adopted, P5 did not significantly affect the FACS signals measured from the coated RBCs. Hence, although of short length, the PEG in P5 played a decisive role in the lack of cell reactivity, in particular, by comparison to P3. We expect that a statistical distribution of the PEG grafts along the PDMAEMA backbone hampers the ionic interaction of the copolymer on the RBC surface. In the case of polymers P3 and $\mathrm{P} 4$, their PEG moieties are sufficiently separated from the polycationic sequence. This pseudoblock copolymer structure is therefore more favorable to anchor the PDMAEMA to the cell plasma membrane while leaving the PEG extremity-free. Clearly, architecture plays a major role in the adsorption of polymer and camouflage ability of RBCs.

These antagonistic behaviors should definitely find their origin in a modification in affinity for those copolymers having 
cell membranes affected by the temperature. From this point of view, it is important to remind that the two polymer sequences belonging to our polycations are well known to be thermoresponsive with a lower critical solution temperature (LCST) in aqueous solution of 30 to $40{ }^{\circ} \mathrm{C}$ and $100-150{ }^{\circ} \mathrm{C}$ for PDMAEMA and PEO, respectively, depending on their $M_{\mathrm{w}}$ and $\mathrm{pH}^{33}$ For example, in the case of PDMAEMA, its full protonation at lower $\mathrm{pH}$ does not allow its phase transition, which is observed only at either neutral or basic $\mathrm{pH}$. Beyond this temperature, these polymers become insoluble in water, a behavior that is typical for the polymers that form hydrogen bonds to water. As a consequence, within this critical temperature zone, the physicochemical state of the macromolecules drastically changes with, as a first step, the conformational change of the molecules that undergoes coilto-globule transition before achieving within a second step the cloud point, which can be macroscopically observed. Definitely through this dehydration process of the polycations coming from the breakage of the hydrogen bond, a decrease in polarity of PDMAEMA should be observed with a temperature increase and should promote their adsorption to RBCs membrane. This change in macromolecule shape and hydrodynamic diameter in this transition regime has been suggested to explain the superior antibacterial activity of a PDMAEMA $\left(M_{\mathrm{w}}-2800\right)$ around $37-43{ }^{\circ} \mathrm{C}$ compared with ambient temperature (adsorption on cell membrane and perturbation). ${ }^{33}$

The temperature of phase transition of our copolymers should be increased by the presence of the PEG grafts compared with the homopolymer of DMAEMA. For example, Ward et al. have reported a cloud point of $72{ }^{\circ} \mathrm{C}$ for PEGMADMAEMA copolymer (with theoretical sequence lengths of 2920-6580, respectively) when dissolved in an aqueous medium at $\mathrm{pH} 7.8 .^{32}$ However, Jean et al. found that the cloud point corresponds to the macroscopic phase separation of thermoresponsive polymers that have already undergone molecular conformational changes at lower temperatures. ${ }^{34}$ In addition to this explanation, which needs support by a physicochemical characterization of our polycation solutions, we cannot rule out a kinetics contribution. Hence, the effect of temperature is clarified because if that factor increases, then the Brownian movement of molecules will also increase, promoting contact between RBCs and PDMAEMAs and resulting in higher polymer adsorption. Considering that temperature increment has been reported as a factor enhancing polycation adsorption, $^{35}$ it might occur.

The effect of temperature on the MFI percentage has also been calculated for samples treated with $100 \mu \mathrm{g} / \mathrm{mL}$, thus showing the highest percentage of pcfRBCs (Figure 7). Interestingly, the copolymers reduced the percentage of MFI to the same extent as that observed for the homopolymers at 10 $\mu \mathrm{g} / \mathrm{mL}$. These observations highlight that the PDMAEMA sequence plays the major contribution in masking antigen effectiveness, whereas the presence of the PEG moieties mostly increases their protection efficiency within a given population of RBCs.

In this respect, the PEG in the PDMAEMA backbone might not have had the physical properties necessary for masking, especially considering the size of the PEG chains. As reported by Bradley et al., antigen size and MW of polymer are important in the efficacy of immunocamouflage. For antigenic determinants of $\mathrm{Jka} / \mathrm{Jkb}$ blood groups farther out on from the cell surface, the MW 5000 PEG was inefficient for masking; but

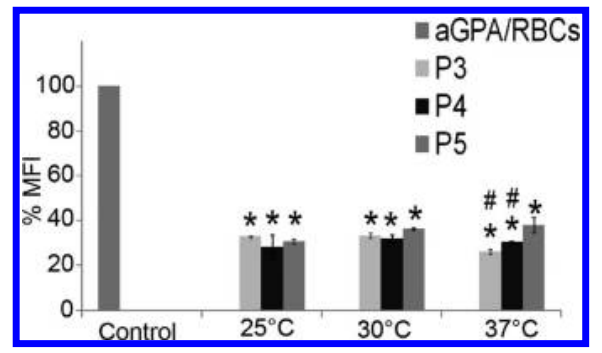

Figure 7. Comparison of the immunomasking effectiveness of the copolymers based on the \% MFI of samples treated with polycations P3, P4, and P5 $(100 \mu \mathrm{g} / \mathrm{mL})$. Samples with $(*)$ showed significant statistically differences respect to the control: RBCs/aGPA MFI \%. Samples with (\#) showed significant statistically differences respect to P5 at $37^{\circ} \mathrm{C}$.

when MW (20000) and density increased, the PEG covered those antigenic sites. ${ }^{36}$

In our case, it was expected that PDMAEMAs exhibit enough volume to cover the GPA extracellular antigenic domain having a MW of 22000 . However, the protection does not depend only on the covered volume. When immunoprotecting the antigen surface, one should consider not only protection from a dimensional standpoint but also the total exclusion volume for diffusing the antibodies according to the volume occupied by the antigenic structures of GPA, but once adsorbed, the polycation molecule may change to conform to a more restricted area of protection. For instance, on inert surfaces, polycation adsorption may take the form of trains or loops, depending on the difference in segment length between both the cationic groups on the polycation backbone and the anionic sites expressed at the surface. ${ }^{33,37}$ If a similar adsorption behavior occurs on the RBC membrane, then we should also take into account the fact that the RBC membrane is a living system wherein the lateral mobility of proteins could significantly influence charge redistribution. ${ }^{38}$ Therefore, polycation adsorption on the RBC surface is not as predictable as that on a static surface. This unpredictable behavior is apparent in our study when we compared the protection effectiveness of the three copolymers. Although P4 presented the longest PEG chains (MW 3050), it did not induce a lowering MFI as it might be expected according to the effect of the PEG MW on camouflaging, as discussed above.

In addition to complexities of polycation-RBC interaction, it should be noted that subject-to-subject variations may affect polycation adsorption and their subsequent effects on the RBC membrane. Even the heterogeneity of a cell population coming from the same blood donor can affect the interaction between RBCs and polycations. For instance, Sovadinova et al. have observed that the effects of amphiphilic methacrylate copolymers containing primary amino groups (MW of copolymers: $2300-2800$ ) on RBCs varied because of cell-tocell differences. ${ }^{39}$ A major finding of that study was that the polycations induced nanoporosity in a heterogeneous pattern from cell to cell. The researchers interpreted their observations as a possible consequence of a variation in polymer adsorption profile among cells due to an erythrocyte-to-erythrocyte variation in sensitivity to pore formation. ${ }^{39}$ In the same context, polymer-induced blood response variations have also been reported by Armstrong et al., in this case regarding PEGand dextran-induced RBC aggregation. ${ }^{40}$ In addition to this interindividual variability, the distribution of erythrocyte ages within a given blood sample with associated biochemical and 
cellular age-dependent variations has been used to explain the unpredictable polymer-induced blood response. ${ }^{40}$ Differences in hemoreactivity to distinct synthetic polymers have been reviewed by Rampling et al., who stressed the need to investigate the sources of those membrane surface variations. ${ }^{41}$

\section{CONCLUSIONS}

In the perspectives of blood transfusion applications, but also drug delivery system, ${ }^{42,43}$ we have compared the camouflaging ability of PDMAEMA homopolymers and PDMAEMA-co-PEG copolymers to cover RBCs. From this comparative study, the following critical clear statements can be drawn.

If a repulsive action could already be expected from RBC's surface modified with PDMAEMA homopolymer, ${ }^{19}$ then only a maximum of $10 \%$ of the whole RBCs were partially (up to $80 \%$ ) masked by the largest PDMAEMA tested while preventing nonspecific cell aggregation. The adoption of higher $M_{\mathrm{w}}$ PDMAEMA should be avoided taking into account the fact that this increase in size is detrimental in hemocompatibility. ${ }^{16}$ Interestingly enough above a $M_{\mathrm{w}}$ of 10000 and a concentrations of $2 \mu \mathrm{g} / \mathrm{mL}$ PDMAMEA selectively enhances cell agglutination following immune recognition of the GPA antigens on the RBC surface, an observation that would merit additional efforts in view to enhance immunodiagnostic recognitions.

As expected, the PEG sequences of the palm-tree architecture enhance the stability of the RBCs and allow us to raise the immunomasking efficiency (up to 40\%) but without, however, achieving a full protection against Anti-GPA recognition (maximum protection effectiveness of $80 \%$ ). The lack of difference in camouflage efficiency between copolymer P3 and P4 suggests that the critical $M_{\mathrm{w}}$ to afford the steric stabilization of RBCs is relatively short $(\sim 480)$, whereas larger polyethylene chains could perhaps contribute to improve their effectiveness, that is, the extent of polymer covering per cell.

If the brush-like comb-polymer architecture of random copolymer P5 could provide better steric performance with a structure theoretically more favorable to stretch the polymer backbone, while leaving the side-chains almost unstretched, then in practice this copolymer would be less efficient than the copolymers and the homopolymers. The random distribution of PEG segments probably best impaired the adsorption of the polycation backbone to the RBC's membrane.

Interestingly enough, a sensitization in aGPA detection has been highlighted for a given fraction of RBCs after incubation with the polycations. This process, temperature-dependent and particularly observed for block-type copolymers, would merit future attention.

\section{AUTHOR INFORMATION}

\section{Corresponding Author}

*E-mail: C.Grandfils@ulg.ac.be. Phone: + 32 (0)4 3663506. Fax: +32 (0) 43663623 .

\section{Notes}

The authors declare no competing financial interest.

\section{ACKNOWLEDGMENTS}

We acknowledge financial support from the EU FP6 IP NanoBioPharmaceutics, Doctorado Institucional en Ingeniería y Ciencia de Materiales-CONACYT México (no. 213606, grant 14781) as well as PIFI 2009 grant.

\section{REFERENCES}

(1) Garratty, G. Vox Sang. 2008, 94, 87-95.

(2) Wilson, J. T.; Krishnamurthy, V. R.; Cui, W.; Qu, Z.; Chaikof, E.

L. J. Am. Chem. Soc. 2009, 131, 18228-18229.

(3) Hosoi, E. J. Med. Invest. 2008, 55, 174-182.

(4) Van Kim, C. L.; Colin, Y.; Cartron, J. P. Blood Rev. 2006, 20, $93-$ 110.

(5) Poole, J.; Daniels, G. Transfus. Med. Rev. 2007, 21, 58-71.

(6) Rudlof, B.; Just, B.; Deitenbeck, R.; Ehmann, Th. Saudi J. Anaesthesia 2011, 5, 101-104.

(7) Kruskall, M. S.; AuBuchon, J. P. Transfus. Sci. 1997, 18, 613-620.

(8) Scott, M. D.; Bradley, A. J.; Murad, K. L. Transfus. Med. Rev. 2000, 14, 53-63.

(9) Olsson, M. L.; Clausen, H. Br. J. Hamaetol. 2008, 140, 3-12.

(10) Olsson, M. L.; Hill, C. A.; de la Vega, H.; Liu, Q. P.; Stroud, M. R.; Valdinocci, J.; Moon, S.; Clausen, H.; Kruskall, M. S. Transfus. Clin. Biol. 2004, 11, 33-39.

(11) Le, Y.; Scott, M. D. Acta Biomater. 2010, 6, 2631-2641.

(12) Mansouri, S.; Fatisson, J.; Miao, Z.; Merhi, Y.; Winnik, F. M.; Tabrizian, M. Langmuir 2009, 25, 14071-14078.

(13) Mansouri, S.; Merhi, Y.; Winnik, F. M.; Tabrizian, M. Biomacromolecules 2011, 12, 585-592.

(14) Elbert, D. L.; Hubbell, J. A. Chem. Biol. 1998, 5, 177-183.

(15) Riquelme, B. D.; Dumas, D.; Fontana, A.; Delannoy, M.; Valverde, J. R.; Sondag, D.; Grandfils, C. J. Biomed. Mater. Res., Part A 2011, 99A, 445-454.

(16) Cerda-Cristerna, B. I.; Flores, H.; Pozos-Guillen, A.; Perez, E.; Sevrin, C.; Grandfils, C. J. Controlled Release 2011, 153, 269-277.

(17) McCarthy, D. A. In Flow Cytometry; Macey, M. G., Ed.; Humana Press: Totowa, NJ, 2007; pp 59-112.

(18) Reid, M. E.; Yahalom, V. Bailliere's Best Pract. Res., Clin. Haematol. 2000, 13, 485-509.

(19) Su, Y. L.; Li, C. J. Colloid Interface Sci. 2007, 316, 344-349.

(20) Grandfils, C.; Foresto, P.; Riquelme, B.; Valverde, J.; SondagThull, D. J. Biomed. Mater. Res., Part A 2008, 84, 535-544.

(21) Mayhew, E.; Harlos, J. P.; Juliano, R. L. J. Membr. Biol. 1973, 14, 213-228.

(22) Moreau, E.; Domurado, M.; Chapon, P.; Vert, M.; Domurad, D. J. Drug Targeting 2002, 10, 161-173.

(23) Moreau, E.; Ferrari, I.; Drochon, A.; Chapon, P.; Vert, M.; Domurado, D. J. Controlled Release 2000, 64, 115-128.

(24) Chasis, J. A.; Mohandas, N. Blood 1992, 80, 1869-1879.

(25) Li, G.; Stewart, R.; Conlan, B.; Gilbert, A.; Roeth, P.; Nair, H. Vox Sang. 2002, 83, 332-338.

(26) Reverberi, R.; Reverberi, L. Blood Transfus. 2007, 5, 227-240.

(27) Lalezari, P.; Jiang, A. F. Transfusion 1980, 20, 206-211.

(28) Fung, Y. Biomechanics. Mechanical Properties of Living Tissues, 2nd ed.; Springer: New York, 1993; pp 113-114.

(29) Kovačević, D.; Glavanović, S.; Peran, N. Colloids Surf., A 2006, 277, 177-182.

(30) Coller, B. Blood 1980, 55, 276-281.

(31) van de Wetering, P.; Zuidam, N. J.; van Steenbergen, M. J.; van der Houwen, O. A. G. J.; Underberg, W. J. M.; Hennink, W. E. Macromolecules 1998, 31, 8063-8068.

(32) Ward, M. A.; Georgiou, T. K. J. Polym. Sci., Part A: Polym. Chem. 2010, 48, 775-783.

(33) Rawlinson, L.-A. B.; Ryan, S. M.; Mantovani, G. Biomacromolecules 2010, 11, 443-453.

(34) Jean, B.; Lee, L.-T.; Cabane, B. Langmuir 1999, 15, 7585-7590.

(35) an, J.-H.; Dultz, S. Clay Miner. 2007, 42, 329-339.

(36) Bradley, A. J.; Murad, K. L.; Regan, K. L.; Scott, M. D. Biochim. Biophys. Acta 2002, 1561, 147-158.

(37) Breen, C. Appl. Clay Sci. 1999, 15, 187-219.

(38) Yawata, Y. Cell Membrane; Wiley-VCH: Weinheim, Germany, 2004; pp 27-46.

(39) Sovadinova, I.; Palermo, E. F.; Huang, R.; Thoma, L. M.; Kuroda, K. Biomacromolecules 2011, 12, 260-268.

(40) Armstrong, J. K.; Wenby, R. B.; Meiselman, H. J.; Fisher, T. C. Biophys. J. 2004, 87, 4259-4270. 
(41) Rampling, M. W.; Meiselman, H. J.; Neu, B.; Baskurt, O. K. Biorheology 2004, 41, 91-112.

(42) Rossi, N. A.; Constantinescu, I.; Kainthan, R. K.; Brooks, D. E.; Scott, M. D.; Kizhakkedathu, J. N. Biomaterials 2010, 31, 4167-78.

(43) Blackall, D. P.; Armstrong, J. K.; Meiselman, H. J.; Fisher, T. C. Blood 2001, 97, 551-556. 